\title{
Bacteroidetes and Firmicutes Drive Differing Microbial Diversity and Community Composition Between Micro-environments in the Bovine Rumen
}

\section{Lee Pinnell}

Texas A\&M University

\section{Arquimides Reyes}

Colorado State University

\section{Cory Wolfe}

Colorado State University

Maggie Weinroth

Colorado State University

Jessica Metcalf

Colorado State University

Robert Delmore

Colorado State University

Keith Belk

Colorado State University

Paul Morley ( $\sim$ pmorley@cvm.tamu.edu )

Texas A\&M University

\section{Terry Engle}

Colorado State University

\section{Research Article}

Keywords: $16 \mathrm{~S}$ rRNA, rumen, cattle, microbiome

Posted Date: February 7th, 2022

DOI: https://doi.org/10.21203/rs.3.rs-1178476/v1

License: (c) (1) This work is licensed under a Creative Commons Attribution 4.0 International License.

Read Full License

Version of Record: A version of this preprint was published at Frontiers in Veterinary Science on May 19th, 2022. See the published version at https://doi.org/10.3389/fvets.2022.897996. 


\section{Abstract}

Ruminants are a critical human food source and are a potentially important source of global methane emissions. Because of their unique digestive physiology, ruminants rely upon a symbiotic relationship with complex and rich microbial communities in the foregut to allow digestion of complex carbohydrates. This study used 16S rRNA gene sequencing to investigate the composition of microbial communities of three rumen micro-environments (free fluid, the fibrous pack, and the rumen mucosa) from cattle fed identical diets. While a phylogenetic core including the most abundant and most common ruminal taxa (members of Bacteroidetes and Firmicutes) existed across micro-environments, their abundances differed significantly, and specific lineages were discriminant of individual micro-environments. Firmicutes, and specifically Ruminococcaceae, Lachnospiraceae, and Clostridium were discriminant of fluid communities, while Bacteroidales and Desulfovibrio were discriminant of mucosa-associated communities. A set of four more diverse lineages were discriminant of pack-associated communities that included Paraprevotellaceae, Succinivibrionaceae, RFP12 (Verrucomicrobia), and Treponema. Our findings indicate that different ecological niches within each micro-environment have resulted in significantly different microbial communities and provide baseline values for the diversity and community structure of microbial communities from rumen fluid, pack, and mucosa that can contextualize the influence of environmental factors such as diet.

\section{Introduction}

Ruminant livestock are a critical source of food for humans worldwide, with the population of cattle alone estimated to be over 1.5 billion (FAO, 2020). Ruminants are also of environmental significance as they release considerable amounts of methane into the atmosphere (McMichael et al., 2007). Characterized by their unique method of plant digestion, ruminants rely on a complex consortium of microorganisms to partially digest plant polysaccharides in their forestomach, or rumen before it enters the glandular "true" stomach. Thus, the microbial community within the rumen plays an essential role in the health of the animal by providing a major source of nutrients (Kamra, 2005), and the microbiota of the rumen are critical to the productivity of this important food source and to potential significance of methane emissions.

The rumen is a rich and diverse microbial ecosystem largely composed of anaerobic bacteria, protozoa, anaerobic fungi, methanogenic archaea, and phage. Bacteria represent the most abundant and diverse taxonomic group, and it is the bacterial members of the rumen microbial community that primarily drive the degradation and fermentation of plant fibers and proteins into digestible compounds such as volatile fatty acids and microbial proteins (Brulc et al., 2009, Deusch et al., 2017). Ruminal archaea are mostly limited to methanogenic members of the phylum Euryarchaeota, and more specifically the classes Methanobacteria, Methanomicrobia, and Thermoplasmata. Typically, ruminal microbial communities are predominately made up of starch and sugar degrading organisms (Deusch et al., 2017), though it is well established that diet exerts a strong influence on ruminal microbial community composition (Pitta et al., 2010, Zhang et al., 2014, Lengowski et al., 2016). Despite this, abundant ruminal taxa are remarkably 
consistent across individual animals regardless of their diet or location. In a meta-analysis characterizing ruminal microbial communities in 742 individual ruminants from across the world, the top 30 most abundant microbial genera were found in over $90 \%$ of samples (Henderson et al., 2015).

The microbiota of the rumen as a whole has been well characterized, but relatively few studies have investigated differences in community structure between ruminal components. While there are a large number of ecological niches available, broadly speaking rumen microorganisms can be free-living or particle-attached in ruminal fluid, attached to the fibrous pack, or attached to the ruminal mucosa (Hungate, 1966). Differences in diversity and community structure have been identified between liquid and solid-associated ruminal communities (Kong et al., 2010, de Menezes et al., 2011, Deusch et al., 2017), but comparisons to mucosa-associated communities have been limited to studies aimed at characterizing diet-induced shifts (AlZahal et al., 2017). Mucosa-associated microbial communities in the rumen have also been described temporally (Guo et al., 2021) and been compared to ruminal packassociated communities (Bolyen et al., 2019), but there is little knowledge about which microbial taxa are discriminant of ruminal fluid, pack, and mucosa-associated communities.

This study utilized $16 \mathrm{~S}$ rRNA gene sequencing to characterize the diversity and composition of microbial communities of different locations within the rumen: within the ruminal fluid, fibrous pack, and those found on the mucosa. It was designed to test if the previously described ruminal phylogenetic core is present across ruminal micro-environments, and to discover which microbial taxa were discriminant of these micro-environments. We hypothesized that core rumen taxa would be present across all three micro-environments, but that the composition of fluid, pack, and mucosa-associated microbial communities would differ.

\section{Results}

Microbial richness and diversity. A comparison of observed ASVs was used to test for differences in richness between the ruminal components, and a comparison of Faith's Phylogenetic Distance (FPD) was used to test for differences in diversity. Microbial communities within the rumen fluid were significantly richer than ruminal pack and mucosa communities, which were similar (Figure 1; PW+BH, $n=12, p<$ 0.05). However, the diversity of ruminal fluid and pack communities were not significantly different, and both were significantly more diverse than mucosal communities (Figure $1 ; \mathrm{PW}+\mathrm{BH}, \mathrm{n}=12, \mathrm{p}<0.05$ ).

Rumen phylogenetic core. Of the 117 genera above the detection threshold of $0.1 \%$ relative abundance, $38(32 \%)$ were present in at least $80 \%$ of samples from all three ruminal components across every individual animal, and thus were considered to represent the phylogenetic core (Figure 2). Of the genera within the phylogenetic core, 29 (76\%) of them belong to the phyla Bacteroidetes or Firmicutes. Genera from these taxa, and more specifically the orders Bacteroidales and Clostridiales, were the most abundant members of the phylogenetic core, with 9 of the top 10 most abundant core genera belonging to these two orders (Figure 2). Interestingly, of the 38 core genera, 33 of them were present in $100 \%$ of all samples. 
Microbial community structure of ruminal components. Differences in overall microbial community structure between the three ruminal components was analyzed using NMDS, hierarchal clustering, and PERMANOVA. The composition of ruminal fluid, pack, and mucosa communities were significantly different from each other when compared using all three UniFrac distances (Table S1; pairwise PERMANOVA, $n=12, p<0.05)$. Non-significant PERMDISP tests, with the exception of pack versus mucosa based on unweighted UniFrac, confirmed that significant PERMANOVA results were the result of differences in community structure and not due to unequal dispersions of variance (Table S1; pairwise PERMDISP, $p>0.05$ ). Visualization with NMDS illustrated that microbial communities from each of the three ruminal components were distinct, and that communities became more similar when compared using less weight on abundant lineages (i.e., from weighted to unweighted UniFrac). Further, fluid and pack communities appear to be more tightly clustered and distinct from each other, while mucosa communities were more varied (Figure 3 ).

Hierarchal clustering based on generalized UniFrac values revealed that fluid communities were most similar to other fluid communities and formed a fluid-specific clade, with the exception of one fluid community that formed a clade with pack and mucosa communities collected from the same individual animal (Figure 4). Ruminal pack and mucosa communities were more intermixed, however both pack and mucosa communities were slightly more likely to cluster with other communities from the same ruminal component (i.e., pack or mucosa). The dichotomy between rumen fluid and pack or mucosa communities was primarily the result in large differences in the abundances of Bacteroidales ( $45 \%$ fluid, $>56 \%$ pack and mucosa) and Clostridiales ( 40\% fluid, $<29 \%$ pack and mucosa) (Figure 4 ).

\section{Ruminal component-specific discriminant lineages}

To more closely investigate taxa that were responsible for the differing community structure between the three ruminal components, linear discriminant analysis effect size (LEfSe) was used to identify taxonomic lineages that were differentially abundant between rumen fluid, pack, and mucosa communities. Results show that 4 lineages of Clostridiales (Ruminococcaceae, Moryella and unassigned Lachnospiraceae, and Clostridium) were discriminant of fluid communities, while Bacteroidales and Desulfovibrio (Desulfovibrionaceae, Desulfovibrionales) were discriminant of mucosa communities (Figure 5). A set of four more diverse lineages including YRCC22 (Paraprevotellaceae), unassigned RFP12 (WCHB1-41, Verrucomicrobia subdivision 5), Treponema (Spirochaetaceae, Spirochaetales), and unassigned Succinivibrionaceae were discriminant of pack communities (Figure 4).

To further characterize the Bacteroidetes-Firmicutes dichotomy between fluid and pack/mucosa samples, the relative abundances of each phylum and the families comprising them were directly compared. The relative abundance of Bacteroidetes significantly differed between each of the three ruminal components, with the phylum being most prevalent in mucosa-associated communities and least prevalent in fluid communities (Figure 6; PW+BH, $n=12, p<0.05$ ). Differences in Bacteroidetes were largely the result of differences within the families of S24-7, Prevotellaceae, and unclassified Bacteroidales (Figure 6; Figure S1). Prevotellaceae and unclassified Bacteroidales were significantly less abundant in fluid communities 
compared to both pack and mucosa, and S24-7 was significantly more abundant in mucosa-associated communities versus both fluid and pack (Figure S1; PW+BH, $n=12, \mathrm{p}<0.05$ ). Interestingly,

Paraprevotellaceae was more abundant in pack communities, although the increased abundance was only significantly then fluid (Figure $\mathrm{S} 1 ; \mathrm{PW}+\mathrm{BH}, \mathrm{n}=12, \mathrm{p}<0.05$ ).

The relative abundance of Firmicutes was significantly different between each of the three ruminal components, with the phylum being significantly more abundant in fluid communities then both pack and mucosa-associated communities, and also significantly less abundant in mucosa-associated communities then pack (Figure 6; $\mathrm{PW}+\mathrm{BH}, \mathrm{n}=12, \mathrm{p}<0.05$ ). The differential abundance of Firmicutes between ruminal components was largely the result of differences in the relative abundance of its most abundant families: Ruminococcaceae, Lachnospiraceae, and unclassified Clostridiales (Figure 6; Figure S2). Lachnospiraceae and unclassified Clostriales were both significantly more abundant in fluid communities then both pack and mucosa-associated communities, while Ruminococcaceae was significantly more abundant in in fluid and mucosa-associated communities then in pack (Figure S2; $\mathrm{PW}+\mathrm{BH}, \mathrm{n}=12, \mathrm{p}<0.05)$.

With the exception the Desulfovibrio lineage, which was discriminant of mucosa-associated communities (Figure 6), every discriminant lineage outside of Firmicutes and Bacteroidetes was from pack-associated communities. Succinivibrionaceae, RFP12, and Spirochaetaceae (Treponema) were all discriminant of pack-associated communities and belong to the phyla Proteobacteria, Verrucomicrobia, and Spirochaetes, respectively (Figure 6). Pack-associated communities had significantly higher relative abundances of Succinivibrionaceae and Spirochaetaceae (virtually entirely made up of Treponema) then fluid and mucosa-associated communities, which had similar abundances (Figure 7; PW+BH, $n=12, p<$ 0.05). The family RFP12 was in significantly higher abundance in pack-associated communities versus both other components and was also significantly higher within fluid communities compared to mucosaassociated communities (Figure 7; $\mathrm{PW}+\mathrm{BH}, \mathrm{n}=12, \mathrm{p}<0.05$ ).

\section{Discussion}

This study used 16S rRNA gene sequencing to investigate the biogeography of microbiota within the bovine rumen and identify microbial taxa discriminant of its micro-environments. Results demonstrated that rumen fluid contained the richest and most diverse microbial communities, while mucosa-associated communities were the least diverse. Importantly, while a large proportion of microbial taxa were present in every ruminal fluid, pack, and mucosa-associated community, the proportions of these taxa differed significantly between ruminal components. Members of the phylum Firmicutes were disproportionately predominant within fluid communities as compared to pack and mucosa-associated communities, while members of the phylum Bacteroidetes were disproportionately under-represented in fluid communities. Further, pack-associated communities contained discriminant lineages from the phyla Bacteroidetes, Proteobacteria, Verrucomicrobia, and Spirochaetes. Diet-induced shifts within rumen microbial communities are well established (Pitta et al., 2010, Zhang et al., 2014, Lengowski et al., 2016, Deusch et al., 2017) and due to the consistent diet throughout this study, these results provide a baseline for the 
diversity and community composition of microbial communities associated with specific ruminal microenvironments (i.e., fluid, pack, and mucosa) that can be used to contextualize the influence of environmental factors such as diet.

Rumen fluid functions as a transportation and growth medium for digestive microorganisms and as a result could be expected to contain the vast majority of microorganisms present in the rumen. Indeed, fluid communities in this study were the richest, but diversity was similar in fluid and pack-associated communities. Higher richness, but similar diversity suggests that ruminal fluid contains more types of microorganisms, but the additional types are either rare or closely related. Interestingly, mucosaassociated communities in this study were both less rich and less diverse, which contradicts previous research that found mucosal communities to be most diverse (AlZahal et al., 2017). However, the previous study performed OTU clustering with an unusually low similarity cutoff (95\%), while this study denoised sequences to produce ASVs without the need to cluster. Denoising provides and a higher resolution look at the community (Callahan et al., 2016), and the differing methodologies likely had a large impact on alpha-diversity metrics and explain the contradictory results.

A large number of studies have used amplicon sequencing to characterize the rumen's phylogenetic core and illustrated the overwhelming dominance of the phyla Bacteroidetes and Firmicutes, and more specifically members of orders Bacteroidiales and Clostridiales (Petri et al., 2014, Henderson et al., 2015, Wirth et al., 2018, Wallace et al., 2019). Here, we demonstrated that these two orders remain very abundant and were found in $100 \%$ of samples collected from ruminal fluid, pack, and mucosa-associated communities. Our finding that $32 \%$ of genera were included in the phylogenetic core across the three micro-environments falls within the range of previous research investigating the ruminal phylogenetic core in whole-rumen samples (Jami \& Mizrahi, 2012, Wirth et al., 2018, Xue et al., 2018). The shared core between each micro-environment is in itself largely uninteresting, as it only illustrates the presence of microbial taxa and not abundance.

Despite the most abundant taxa being dominant in all three micro-environments, fluid, pack, and mucosaassociated microbial communities were different when compared with and without weight being placed on abundance, suggesting that both abundant and rare taxa contribute to the differences. Variations in community composition in communities associated with liquid- and solid-fractions of the rumen different ruminal micro-environments have been well described (Kong et al., 2010, AlZahal et al., 2017, Deusch et al., 2017) and this study confirms this. Further, our results illustrate that the difference between liquidand solid-associated fractions is much larger than that between solid- and mucosa-associated communities. The dichotomy between free-living and attached microbial communities is a longestablished fact in environmental microbial ecology (DeLong et al., 1993, Crump et al., 1999, Hollibaugh et al., 2000, Liu et al., 2019) and here we demonstrate this likely holds true in the rumen as well.

The primary drivers of the liquid-solid dichotomy in the rumen were members of the phyla Bacteroidetes and Firmicutes. In particular, the abundances of unclassified members of the order Bacteroidales and the family Prevotellaceae were significantly more abundant in fluid communities, while unclassified members 
of Clostridiales, Ruminococcaceae, and Lachnospiraceae were significantly more abundant in solidattached communities. Indeed, all four lineages discriminant of fluid communities were members of Firmicutes, and the order Bacteroidales was discriminant of mucosa-associated samples. A largely undescribed genus closely related to members of Prevotellaceae, $Y R C 22$, was discriminant of packassociated communities. YRC22 has been described in numerous studies investigating ruminal microbial communities (McCann et al., 2016, Tao et al., 2017, Jin et al., 2018) but further work is required to understand its function. Our results suggest it likely plays a larger role in ruminal content-attached communities. Previous results have been mixed in illustrating differences in the abundance of Bacteroidetes and Firmicutes between liquid and solid rumen communities; either reporting very small differences between fluid and solids (Deusch et al., 2017), or results similar to ours (AlZahal et al., 2017).

It should also be noted that the samples were collected from cattle that had permanent fistulas placed through the skin into the rumen. While a plugged canula was maintained in the fistulas throughout this study, except at the time of sampling, this opening had the potential to alter the rumen environment because of increased exposure to atmospheric gases. Accessing the rumen via these fistulas also had the potential to introduce microbiota, potentially altering the normal flora of the rumen, albeit minimally. However, it is unlikely that these factors would have been fully responsible for creating systematic differences among the microbiota found in the different ruminal fractions that were study. One additional potentially confounding factor in this study was the use of different extraction methods for fluid and solid communities (pack and mucosa-associated), which has been demonstrated to influence 16S rRNA amplicon sequencing and subsequent community composition results in ruminal communities (Henderson et al., 2013, Villegas-Rivera et al., 2013). However, while two extraction kits were used, they both used mechanical shearing (bead-beating) without phenol and both kits utilize the same chemistries, which eliminates the largest sources of extraction bias (Henderson et al., 2013). As a result, there was likely minimal influence on apparent differences in microbial community structure.

Multiple lineages outside Bacteroidetes and Firmicutes were also discriminant of individual microenvironments. With the exception of Desulfovibrio, a common sulfate-reducing microorganism that was discriminant of mucosa-associated communities, all lineages outside the two major phyla were discriminant of pack-associated communities. However, like many members of the ruminal microbial community, further work is needed to characterize its role. Members of Treponema are known to be involved with the degradation of soluble fibers (Bekele et al., 2011), and it follows they, along with their family Spirochaetaceae and order Spirochaetales, would be over-represented in communities attached to the fibrous pack. Members of the family Succinivibrionaceae ferment carbohydrate to produce succinate and acetate (Xue et al., 2019) and were also was discriminant of pack-associated communities. Interestingly, this family has been linked to lower methane emissions (Wallace et al., 2015). Contrastingly, the family RFP12 from the phylum Verrucomicrobia was discriminant of pack-associated communities but has been negatively correlated with lower methane emissions (Cunha et al., 2017). Our results suggest that efforts to promote or negate the growth of these families implicated in methane emissions should potentially target the flora that are abundant in the ruminal fibrous pack. 
Our results demonstrated that while a dominant phylogenetic core exists across rumen microenvironments (i.e., fluid, pack, mucosa) the proportions of these taxa differed significantly between ruminal components. Members of Firmicutes were predominant within fluid communities, while members of the phylum Bacteroidetes were disproportionately dominant in attached communities. Further, packassociated communities contained discriminant lineages from the phyla Bacteroidetes, Proteobacteria, Verrucomicrobia, and Spirochaetes. These results provide a baseline for the diversity and composition of microbial communities associated with specific ruminal micro-environments and provide critical information on which ruminal components future studies should target in their efforts to alter rumen microbiota for the purposes of both feed efficiency and reduced methane emissions.

\section{Methods}

Cattle Population. Twelve crossbred feedlot steers ( $450 \mathrm{~kg} ; ~ 3.0$ years of age) fitted with ruminal fistulas, were utilized in this study. All cattle in this study were adjusted to a high energy finishing diet consisting of $90 \%$ concentrate and $10 \%$ roughage $(1.43 \mathrm{NEg}, \mathrm{Mcal} / \mathrm{kg})$ for 4 weeks $(28 \mathrm{~d})$ before sample collection. Animals were managed and sampled in accordance with Colorado State University's (CSU) Institutional Animal Care and Use Committee (IACUC) approval (Protocol 16-6550A). Steers were housed at CSU's Agricultural Research, Development and Education Center.

Rumen Content Collection. At the completion of the diet adjustment period, samples from three microenvironments within the rumen were collected via ruminal fistulas from each steer approximately $1 \mathrm{~h}$ after morning feeding: the aqueous portion of the rumen (fluid; $n=12$ ), a bolus of the ruminal fibrous pack (pack; $n=12$ ), and a swab of the ruminal mucosa (mucosa; $n=12$ ). The bolus of fibrous material was sampled by collecting $85 \mathrm{~g}$ from the center of the solid portion of the rumen, and the ruminal mucosa was sampled by running a sterile swab across the ruminal wall. Fluid was collected by compressing the fibrous bolus and capturing the resulting liquid. After collection, samples were immediately placed on ice, transported to the laboratory and stored at $-80^{\circ} \mathrm{C}$ at the laboratory until DNA isolation.

DNA Isolation, 16S rRNA library preparation and sequencing. Genomic DNA was isolated from fluid and mucosal swab samples using a QIAamp PowerFecal DNA Kit (Qiagen, Hilden, Germany) from 0.16 - 0.18 $\mathrm{g}$ of material, while a DNeasy PowerMax Soil Kit was used to isolate DNA from from 2 - $5 \mathrm{~g}$ fibrous pack samples. Following isolation, DNA was quantified $\left(n g \mu L^{1}\right)$ and assayed for quality $\left(A_{260} / A_{280}\right)$ using a NanoDrop spectrophotometer (Thermo Fisher Scientific, Inc., Waltham, MA).

Amplicon library preparation and sequencing was carried out by Novogene Corporation Inc (Chula Vista, CA, USA). The V4 region of the 16S rRNA gene was amplified with the 515f ( $5^{\prime}-\mathrm{GTG}$ CCA GCM GCC GCG GTA A - 3') and 806r (5' - GCA CTA CHV GGG TWT CTA AT - 3') primer pair and Novogene's proprietary amplification conditions. Following successful PCR, amplicon libraries were prepared and pooled using Novogene's proprietary process and sequenced on an Illumina HiSeq 2500 instrument (Illumina Inc., San Diego, CA, USA) using $2 \times 250$ bp paired-end chemistry. The number of sequenced reads ranged from 
188,276 to 219,029 , with an average of 209,279 reads per sample. The average sequencing depth did not differ between the three micro-environments (Pairwise Wilcoxon rank-sum + Benjamini-Hochberg correction for multiple comparisons, $p>0.05, n=12$ ).

Bioinformatics. Demultiplexed paired-end reads were imported into QIIME2 version 2020.11 (Bolyen et al., 2019) and DADA2 was used to filter reads for quality, remove chimeric sequences, merge overlapping paired-end reads, and generate amplicon sequence variants (ASVs) (Callahan et al., 2016). Forward and reverse reads were trimmed at $6 \mathrm{bp}$ and truncated at $247 \mathrm{bp}$ and $250 \mathrm{bp}$ (forward and reverse reads, respectively). Taxonomy was assigned using a Naïve Bayes classifier trained on the Greengenes version 13_8 99\% OTUs database (DeSantis et al., 2006), where sequences had been trimmed to include only the base pairs from the V4 region bound by the $515 f / 806 r$ primer pair. Reads that mapped to chloroplast and mitochondrial sequences were filtered from the representative sequences and ASV table using the 'filter_taxa' function, and a midpoint-rooted phylogenetic tree was then generated using the 'q2-phylogeny' pipeline with default settings, which was used to calculate phylogeny-based diversity metrics. Data and metadata were then imported into phyloseq (McMurdie \& Holmes, 2013) using the 'import_biom' and 'import_qiime_sample_data' functions and merged into a phyloseq object. Richness (Observed ASVs) and Faith's Phylogenetic Diversity (FPD) were calculated for all samples with phyloseq and the 'estimate_pd' function from the btools package. Samples were then proportionally transformed to the lowest total ASV count of 47,420 and beta-diversity was analyzed using weighted, generalized, and unweighted UniFrac distances (Lozupone et al., 2011, Chen et al., 2012). From these distances, non-metric multidimensional scaling (NMDS) was performed and plotted, and a permutational multivariate analysis of variance (PERMANOVA) was used to test for significant differences in community structure using the vegan (Oksanen et al., 2019) and pairwiseAdonis (Arbizu, 2017) packages. To ensure significant differences were not the result of unequal dispersion of variability between groups, permutational analyses of dispersion (PERMDISP) were conducted for all significant PERMANOVA outcomes using the vegan package. Hierarchal clustering was performed using Ward's agglomeration clustering method (Murtagh \& Legendre, 2014) on generalized UniFrac distances and the 'hclust' function. Further, the relative abundances of ASVs within each sample were calculated and plotted using phyloseq.

Rumen phylogenetic core and discriminant lineages. The core microbiota of the rumen was identified at the taxonomic rank of genus. A detection limit (minimum allowed relative abundance) of $0.1 \%$ and a minimum prevalence (proportion of samples the genus was present in) of $80 \%$ were used as cutoffs, and the 'core' function within the package microbiome was used to calculate and plot a heatmap of core community members.

To identify taxa that discriminant of each micro-environment (i.e., fluid, pack, mucosa), linear discriminant analysis effect size (LEfSe) was performed using the online LEfSe tool on the Galaxy server (https://huttenhower.sph.harvard.edu/galaxy) under default settings. Genera with a mean relative abundance $>0.1 \%$ across all samples were considered, with the factor 'micro-environment' set as class, and an "all-against-all" strategy was applied. The relative abundances of the lineages considered discriminant of any of the three micro-environments were then visualized and compared. 
Statistical analyses. Unless specified otherwise, R version 3.6.3 (R Core Team, 2017) was used for statistical analysis of data. Pairwise Wilcoxon rank-sum tests were performed with a Benjamini-Hochberg correction $(\mathrm{PW}+\mathrm{BH})$ for multiple comparisons. Differences in beta diversity were tested using pairwise PERMANOVA with a Benjamini-Hochberg correction for multiple comparisons and 9999 permutations. Additionally, pairwise PERMDISPs were carried out for all significant PERMANOVA outcomes using 9999 permutations to test for differences in the variability of dispersions.

\section{Declarations}

\section{Ethics Approval.}

Animals were managed and sampled in accordance with Colorado State University's (CSU) Institutional Animal Care and Use Committee (IACUC) approval (Protocol 16-6550A). This study was carried out in compliance with ARRIVE guidelines.

\section{Consent for publication.}

NA

\section{Data availability.}

All sequence reads were made available through the BioProject PRJNA749669 at the NCBI's Sequence Read Archive.

\section{Competing Interests.}

The authors declare no competing interests.

\section{Funding.}

This research was funded by MicroBios Inc.

\section{Author's Contributions.}

$\mathrm{KB}, \mathrm{PM}, \mathrm{TE}, \mathrm{JM}, \mathrm{RD}, \mathrm{MW}$, and AR participated in and provided oversight of all aspects of the study including securing funding, design, laboratory procedures, data analysis, and report preparation. $\mathrm{CW}$ participated in study conduct and laboratory analyses. LP performed the data analysis and wrote the manuscript. All authors read, edited, and approved the final manuscript.

\section{Acknowledgements.}

NA

\section{References}


1. AlZahal O, Li F, Guan LL, Walker ND \& McBride BW (2017) Factors influencing ruminal bacterial community diversity and composition and microbial fibrolytic enzyme abundance in lactating dairy cows with a focus on the role of active dry yeast. J Dairy Sci 100: 4377-4393.

2. Arbizu PM (2017) pairwiseAdonis: pairwise multilevel comparison using adonis. p.^pp. https://github.com/pmartinezarbizu/pairwiseAdonis.

3. Bekele AZ, Koike S \& Kobayashi Y (2011) Phylogenetic diversity and dietary association of rumen Treponema revealed using group-specific 16S rRNA gene-based analysis. FEMS Microbiol Lett 316: 51-60.

4. Bolyen E \& Rideout JR \& Dillon MR, et al. (2019) Reproducible, interactive, scalable and extensible microbiome data science using QIIME 2. Nat Biotechno/ 37: 852-857.

5. Brulc JM, Antonopoulos DA, Miller MEB, et al. (2009) Gene-Centric Metagenomics of the FiberAdherent Bovine Rumen Microbiome Reveals Forage Specific Glycoside Hydrolases. Proc Natl Acad Sci U S A 106: 1948-1953.

6. Callahan BJ, McMurdie PJ, Rosen MJ, Han AW, Johnson AJ \& Holmes SP (2016) DADA2: Highresolution sample inference from Illumina amplicon data. Nat Methods 13: 581-583.

7. Chen J, Bittinger K, Charlson ES, Hoffmann C, Lewis J, Wu GD, Collman RG, Bushman FD \& Li H (2012) Associating microbiome composition with environmental covariates using generalized UniFrac distances. Bioinformatics 28: 2106-2113.

8. Crump BC, Armbrust EV \& Baross JA (1999) Phylogenetic analysis of particle-attached and free-living bacterial communities in the Columbia River, its estuary, and the adjacent coastal ocean. Applied and Environmental Microbiology 65: 3192-3204.

9. Cunha CS, Veloso CM, Marcondes MI, Mantovani HC, Tomich TR, Pereira LGR, Ferreira MFL, DillMcFarland KA \& Suen G (2017) Assessing the impact of rumen microbial communities on methane emissions and production traits in Holstein cows in a tropical climate. Systematic and Applied Microbiology 40: 492-499.

10. de Menezes AB, Lewis E, O'Donovan M, O'Neill BF, Clipson N \& Doyle EM (2011) Microbiome analysis of dairy cows fed pasture or total mixed ration diets. FEMS Microbiol Ecol 78: 256-265.

11. DeLong EF, Franks DG \& Alldredge AL (1993) Phylogenetic diversity of aggregate-attached vs. freeliving marine bacterial assemblages. Limnol Oceanogr 38: 924-934.

12. DeSantis TZ, Hugenholtz P, Larsen N, Rojas M, Brodie EL, Keller K, Huber T, Dalevi D, Hu P \& Andersen GL (2006) Greengenes, a Chimera-Checked 16S rRNA Gene Database and Workbench Compatible with ARB. Applied and Environmental Microbiology 72: 5069-5072.

13. Deusch S, Camarinha-Silva A, Conrad J, Beifuss U, Rodehutscord M \& Seifert J (2017) A Structural and Functional Elucidation of the Rumen Microbiome Influenced by Various Diets and Microenvironments. Front Microbio/ 8.

14. FAO (2020) World Food and Agriculture - Statistical Yearbook 2020. p.^pp. 366. Food and Agriculture Organization of the United Nations, Rome, Italy. 
15. Guo W, van Niekerk JK, Zhou M, Steele MA \& Guan LL (2021) Longitudinal assessment revealed the shifts in rumen and colon mucosal-attached microbiota of dairy calves during weaning transition. $J$ Dairy Sci 104: 5948-5963.

16. Henderson G, Cox F, Kittelmann S, Miri VH, Zethof M, Noel SJ, Waghorn GC \& Janssen PH (2013) Effect of DNA Extraction Methods and Sampling Techniques on the Apparent Structure of Cow and Sheep Rumen Microbial Communities. PLoS One 8: e74787.

17. Henderson G \& Cox F \& Ganesh S, et al. (2015) Rumen microbial community composition varies with diet and host, but a core microbiome is found across a wide geographical range. Sci Rep 5: 14567.

18. Hollibaugh JT, Wong PS \& Murrell MC (2000) Similarity of particle-associated and free-living bacterial communities in northern San Francisco Bay, California. Aquat Microb Ecol 21: 103-114.

19. Hungate RE (1966) The rumen and its microbes. Academic Press, New York, NY.

20. Jami E \& Mizrahi I (2012) Composition and Similarity of Bovine Rumen Microbiota across Individual Animals. PLoS One 7: e33306.

21. Jin W, Wang Y, Li Y, Cheng Y \& Zhu W (2018) Temporal changes of the bacterial community colonizing wheat straw in the cow rumen. Anaerobe 50: 1-8.

22. Kamra DN (2005) Rumen microbial ecosystem. Curr Sci 89: 124-135.

23. Kong Y, Teather R \& Forster R (2010) Composition, spatial distribution, and diversity of the bacterial communities in the rumen of cows fed different forages. FEMS Microbiol Ecol 74: 612-622.

24. Lengowski MB, Witzig M, Möhring J, Seyfang GM \& Rodehutscord M (2016) Effects of corn silage and grass silage in ruminant rations on diurnal changes of microbial populations in the rumen of dairy cows. Anaerobe 42: 6-16.

25. Liu K, Hou J, Liu Y, Hu A, Wang M, Wang F, Chen Y \& Gu Z (2019) Biogeography of the free-living and particle-attached bacteria in Tibetan lakes. FEMS Microbiol Ecol 95.

26. Lozupone C, Lladser ME, Knights D, Stombaugh J \& Knight R (2011) UniFrac: an effective distance metric for microbial community comparison. ISME J 5: 169-172.

27. McCann JC, Luan S, Cardoso FC, Derakhshani H, Khafipour E \& Loor JJ (2016) Induction of Subacute Ruminal Acidosis Affects the Ruminal Microbiome and Epithelium. Front Microbio/ 7.

28. McMichael AJ, Powles JW, Butler CD \& Uauy R (2007) Food, livestock production, energy, climate change, and health. The Lancet 370: 1253-1263.

29. McMurdie PJ \& Holmes S (2013) phyloseq: an R package for reproducible interactive analysis and graphics of microbiome census data. PLoS One 8: e61217.

30. Murtagh F \& Legendre P (2014) Ward's hierarchical agglomerative clustering method: which algorithms implement Ward's criterion? J Classif 31: 274-295.

31. Oksanen J, Blanchet FG, Friendly M, et al. (2019) vegan: Community Ecology Package. p.^pp. https://CRAN.R-project.org/package=vegan.

32. Petri RM, Schwaiger T, Penner GB, Beauchemin KA, Forster RJ, McKinnon JJ \& McAllister TA (2014) Characterization of the Core Rumen Microbiome in Cattle during Transition from Forage to 
Concentrate as Well as during and after an Acidotic Challenge. PLoS One 8: e83424.

33. Pitta DW, Pinchak WE, Dowd SE, et al. (2010) Rumen Bacterial Diversity Dynamics Associated with Changing from Bermudagrass Hay to Grazed Winter Wheat Diets. Microb Ecol 59: 511-522.

34. R Core Team (2017) R: A language and environment for statistical computing. p.^pp. R Foundation for Statistical Computing, Vienna, Austria.

35. Tao S, Tian P, Luo Y, Tian J, Hua C, Geng Y, Cong R, Ni Y \& Zhao R (2017) Microbiome-Metabolome Responses to a High-Grain Diet Associated with the Hind-Gut Health of Goats. Front Microbio/ 8.

36. Villegas-Rivera G, Vargas-Cabrera Y, González-Silva N, Aguilera-García F, Gutiérrez-Vázquez E, BravoPatiño A, Cajero-Juárez M, Baizabal-Aguirre VM \& Valdez-Alarcón JJ (2013) Evaluation of DNA extraction methods of rumen microbial populations. World Journal of Microbiology and Biotechnology 29: 301-307.

37. Wallace RJ, Rooke JA, McKain N, Duthie C-A, Hyslop JJ, Ross DW, Waterhouse A, Watson M \& Roehe $\mathrm{R}$ (2015) The rumen microbial metagenome associated with high methane production in cattle. $B M C$ Genomics 16: 839.

38. Wallace RJ, Sasson G, Garnsworthy PC, et al. (2019) A heritable subset of the core rumen microbiome dictates dairy cow productivity and emissions. Sci Adv 5: eaav8391.

39. Wirth R, Kádár G, Kakuk B, Maróti G, Bagi Z, Szilágyi Á, Rákhely G, Horváth J \& Kovács KL (2018) The Planktonic Core Microbiome and Core Functions in the Cattle Rumen by Next Generation Sequencing. Front Microbio/ 9.

40. Xue M, Sun H, Wu X, Guan LL, Liu J \& Nojiri H (2018) Assessment of Rumen Microbiota from a Large Dairy Cattle Cohort Reveals the Pan and Core Bacteriomes Contributing to Varied Phenotypes. Applied and Environmental Microbiology 84: e00970-00918.

41. Xue MY, Sun HZ, Wu XH, Guan LL \& Liu JX (2019) Assessment of rumen bacteria in dairy cows with varied milk protein yield. J Dairy Sci 102: 5031-5041.

42. Zhang R, Zhu W, Zhu W, Liu J \& Mao S (2014) Effect of dietary forage sources on rumen microbiota, rumen fermentation and biogenic amines in dairy cows. Journal of the Science of Food and Agriculture 94: 1886-1895.

\section{Figures}



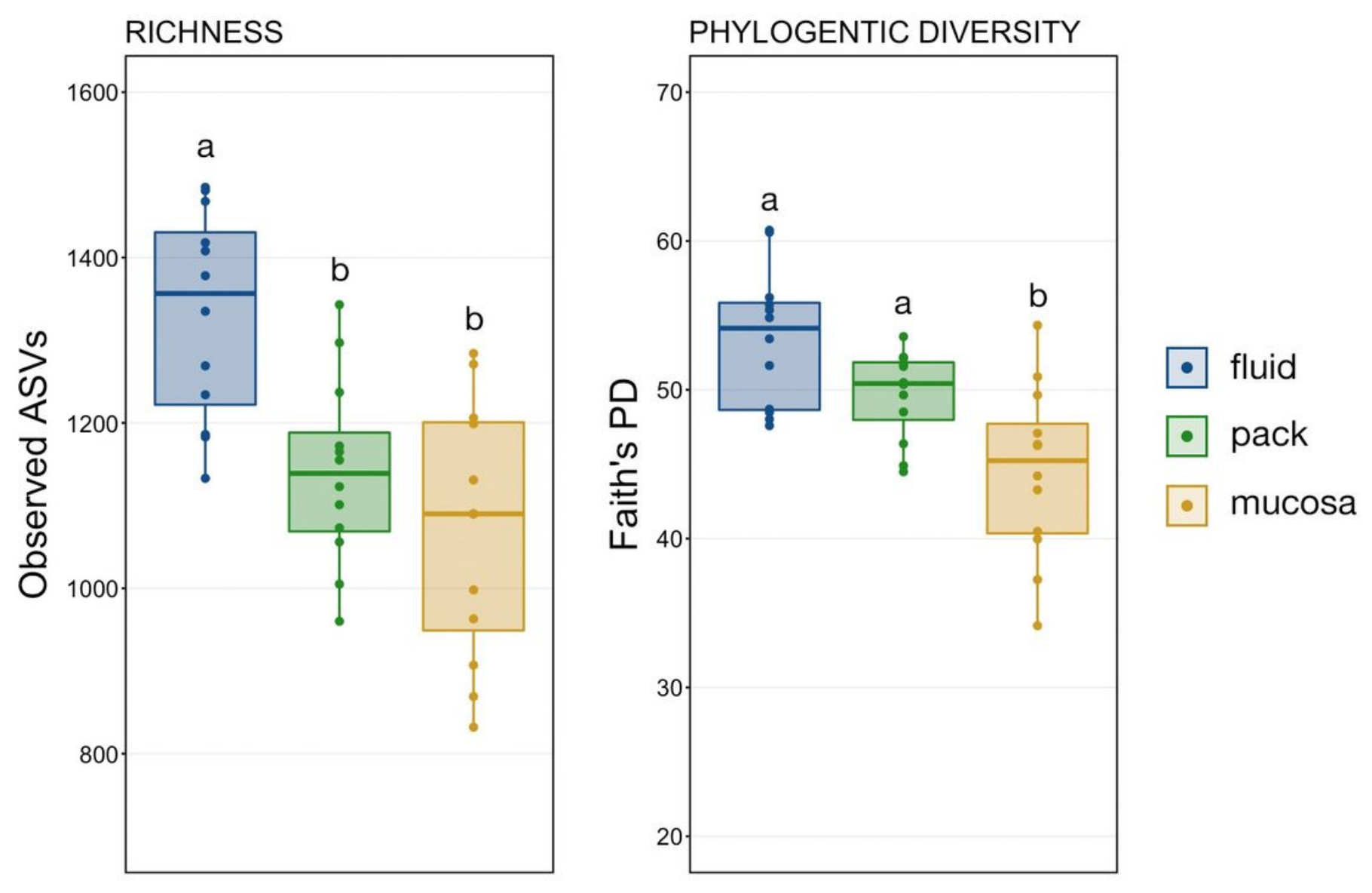

\section{Figure 1}

Boxplots displaying the number of observed ASVs and Faith's Phylogenetic Distance for each ruminal component (fluid, pack, mucosa). Significant differences in richness and diversity between components are illustrated by different letters (Pairwise Wilcoxon rank-sum with Benjamini-Hochberg correction, $\mathrm{p}<$ $0.05, \mathrm{n}=12$ ). 


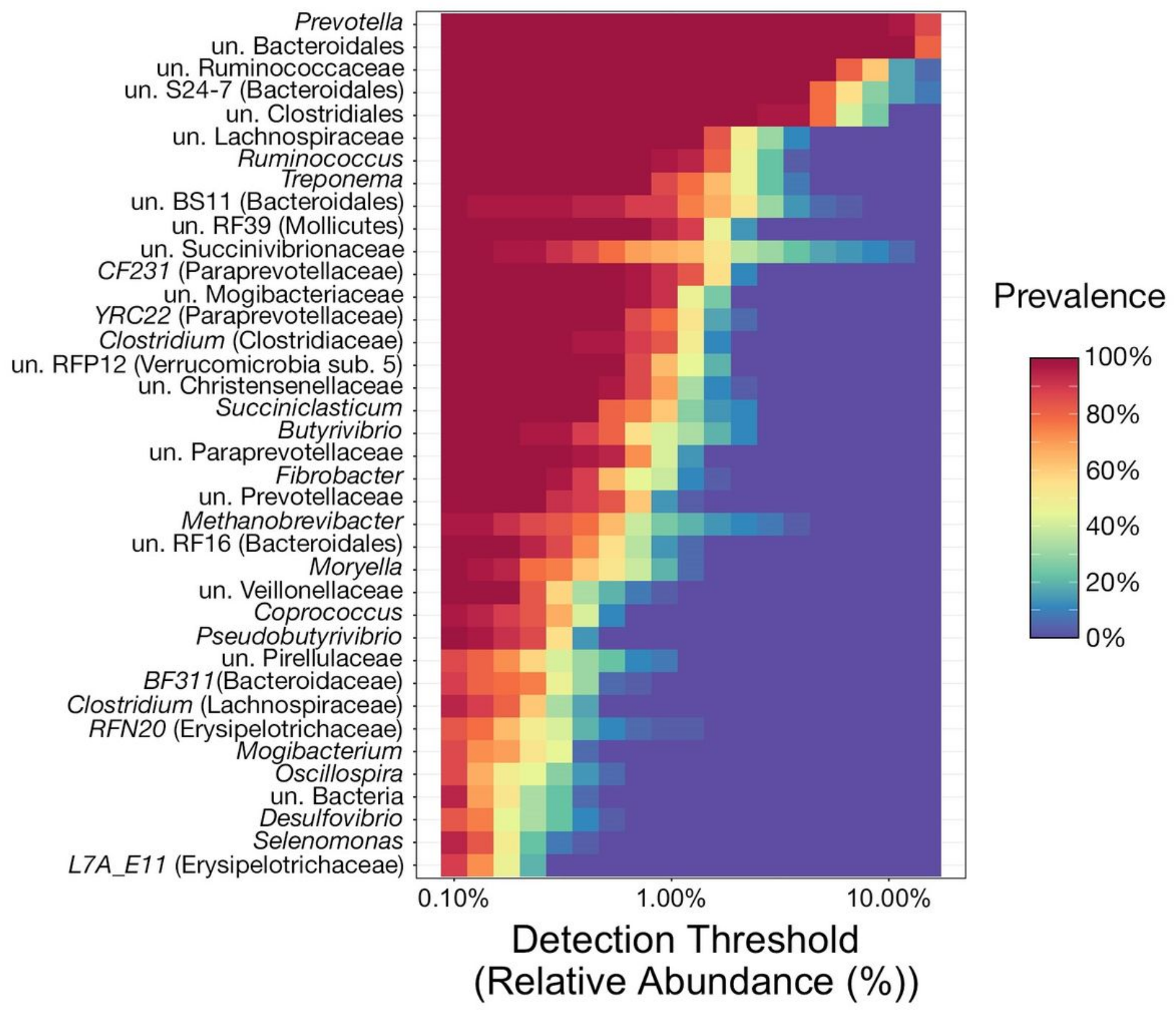

Figure 2

Heatmap illustrating the microbial phylogenetic core of the bovine rumen in feedlot cattle at the taxonomic rank of genus. To be considered core taxa, genera needed to comprise more than $0.1 \%$ of the overall microbial community and be present in at least $80 \%$ of all samples $(n=36)$. The most abundant and most prevalent taxa are at the top of the heatmap, and the least abundant and least prevalent taxa that met the thresholds are at the bottom. A total of 38 genera were considered core taxa. Abbreviations: un., unclassified 
weighted UniFrac

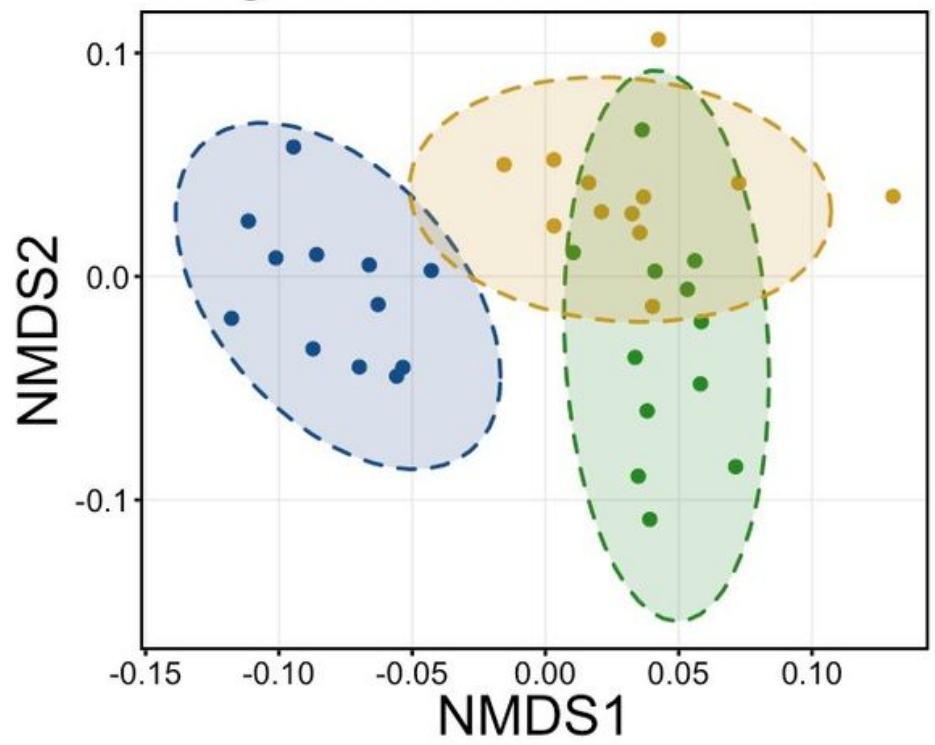

\section{unweighted UniFrac}

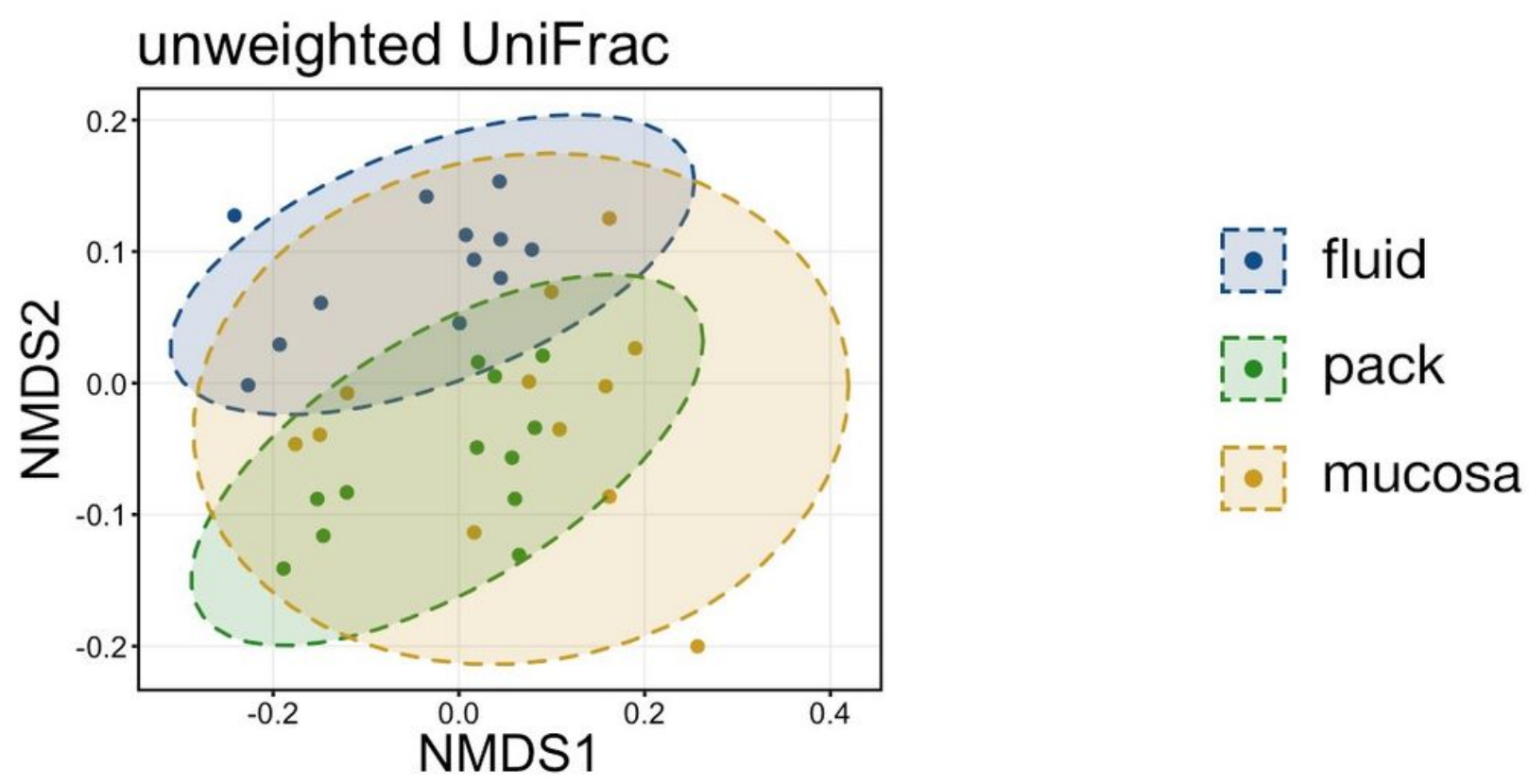

generalized UniFrac

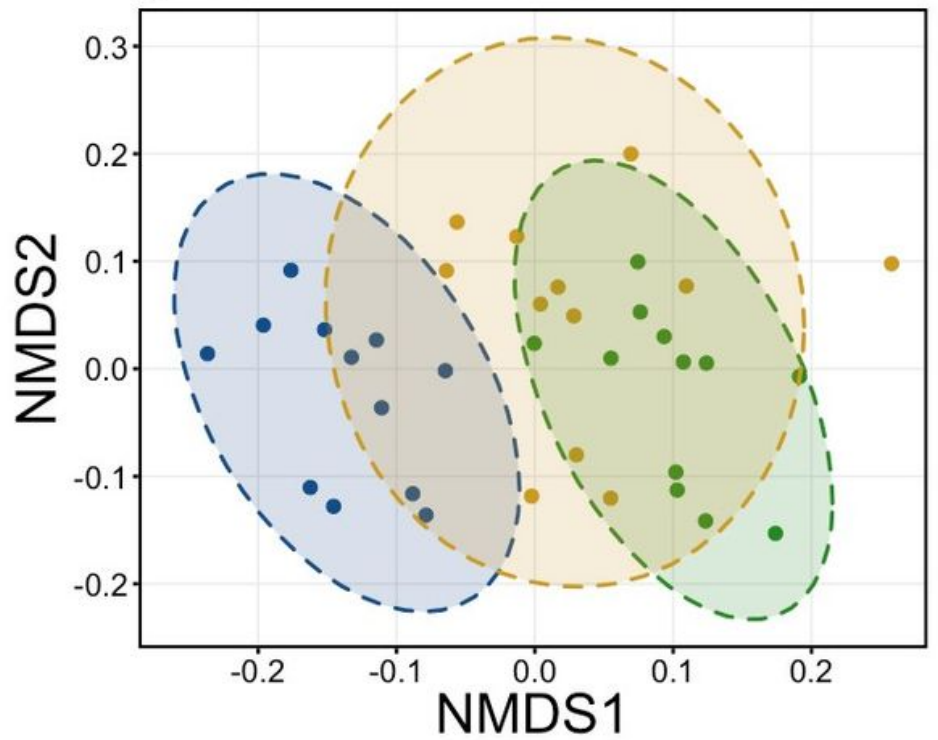

Figure 3

Non-metric multidimensional scaling (NMDS) of weighted, generalized, and unweighted UniFrac distances illustrating variation in microbial community structure associated with each ruminal component. The NMDS demonstrates clustering of 16S rRNA gene sequences from rumen fluid, pack, and mucosal microbial communities. Dashed lines and shaded areas represent $95 \%$ confidence ellipses for each ruminal component. 

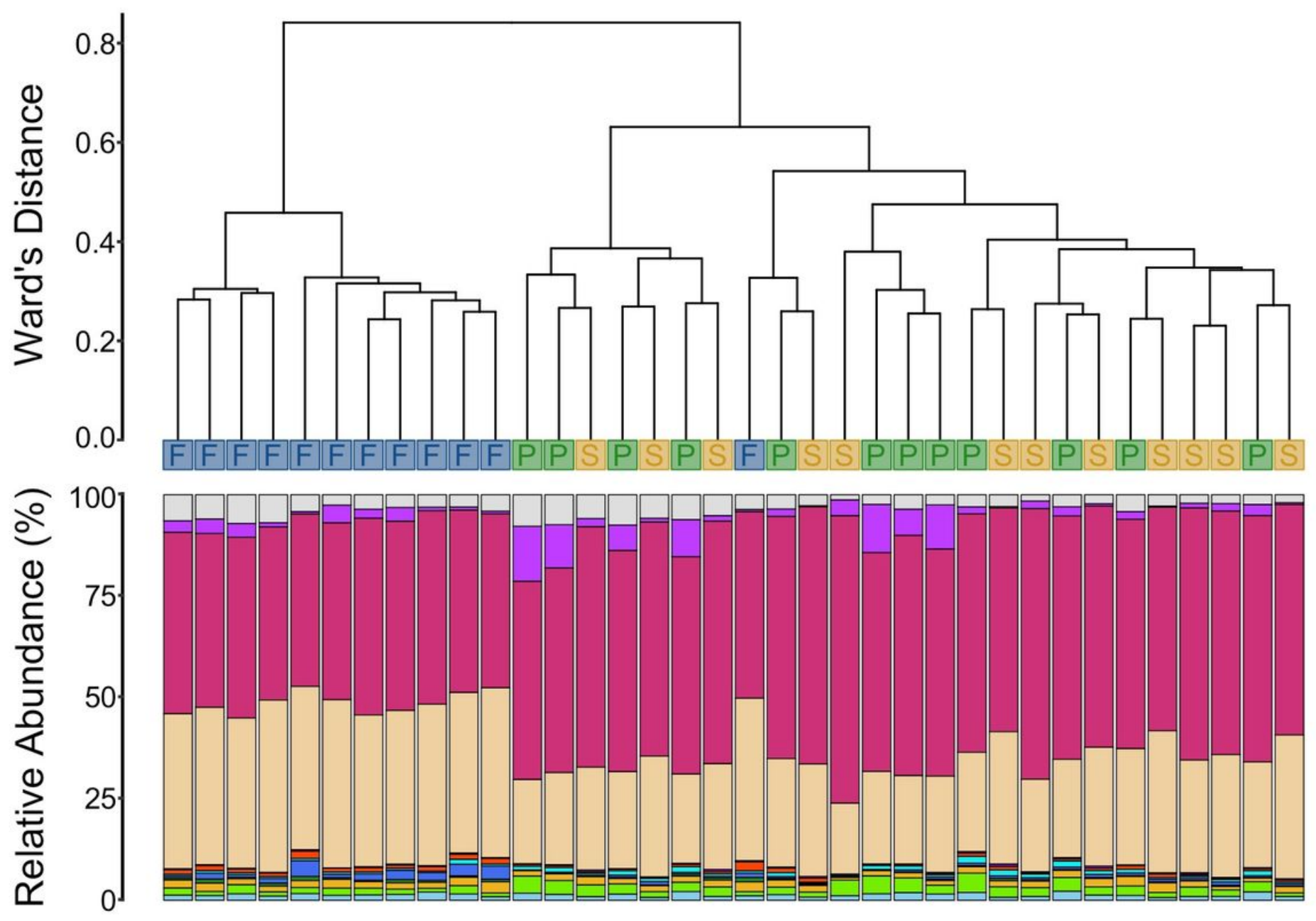

Bacteroidales $\square$ Clostridiales $\square$ Aeromonadales $\square$ Spirochaetales $\square$ RF-39 (Tenericutes) WCHB1-41 (Verrucomicrobia) $\square$ Methanobacteriales $\square$ other $(<0.25 \%)$

\section{Figure 4}

The relatedness of rumen fluid, pack, and mucosa microbial communities based on normalized ASVs. Hierarchal clustering was performed on generalized UniFrac distances using Ward's agglomeration method. Blue boxes $(F)$ represent fluid communities, green boxes $(P)$ represent pack communities, and gold boxes (S) represent mucosal communities. The barplot illustrates the relative abundance of microbial orders within each individual sample. The 8 most abundant orders across all samples are displayed in the legend. 


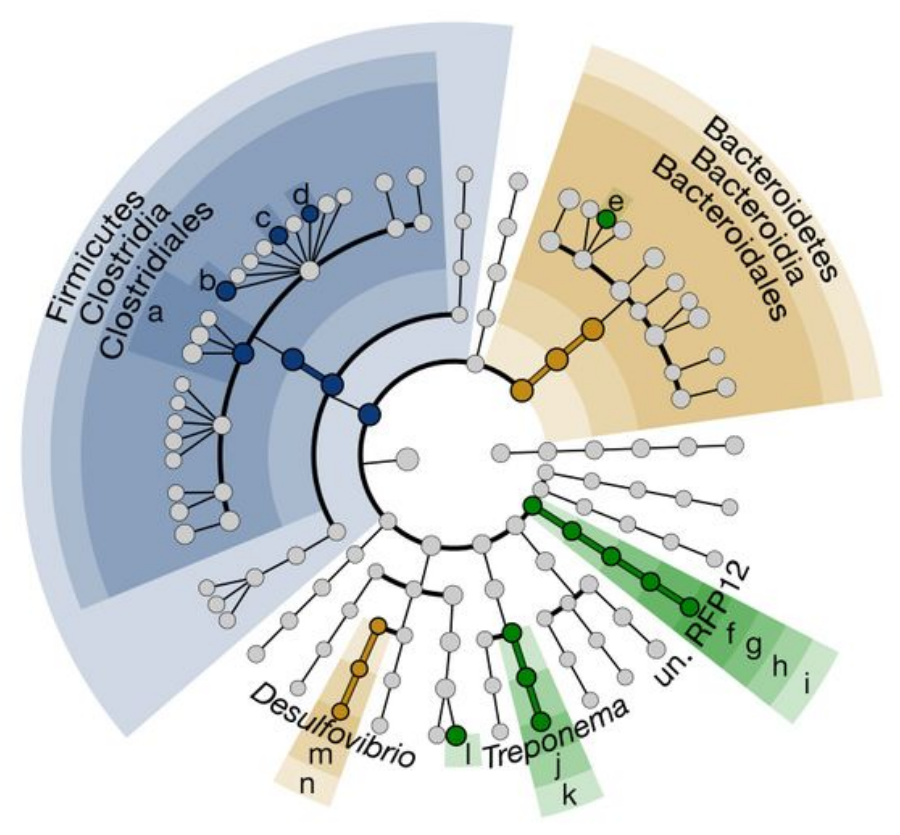

a: Ruminococcaceae
b: un. Lachnospiraceae
c: Moryella
d: Clostridium
e: YRC22 (Paraprevotellaceae)

f-i: RFP12; WCHB1-41; sub. 5; Verrucomicrobia j-k: Spirochaetaceae; Spirocaetales

I: un. Succinivibrionaceae

m-n: Desulfovibrionaceae; Desulfovibrionales

\section{fluid $\bigcirc$ pack mucosa}

\section{Figure 5}

Cladogram demonstrating the microbial taxa with relative abundances of greater than $0.1 \%$ of the total community across fluid, pack, and mucosa-associated microbial communities. Taxa discriminant of fluid communities are highlighted in blue, taxa discriminant of pack communities are highlighted in green, and taxa discriminant of mucosa communities are highlighted in gold (LEfSe, $p<0.05, n=12$ ).
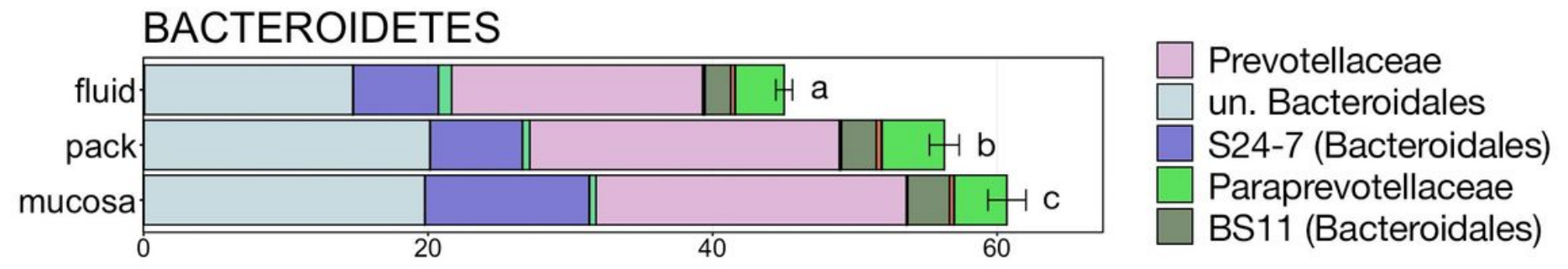

\section{FIRMICUTES}

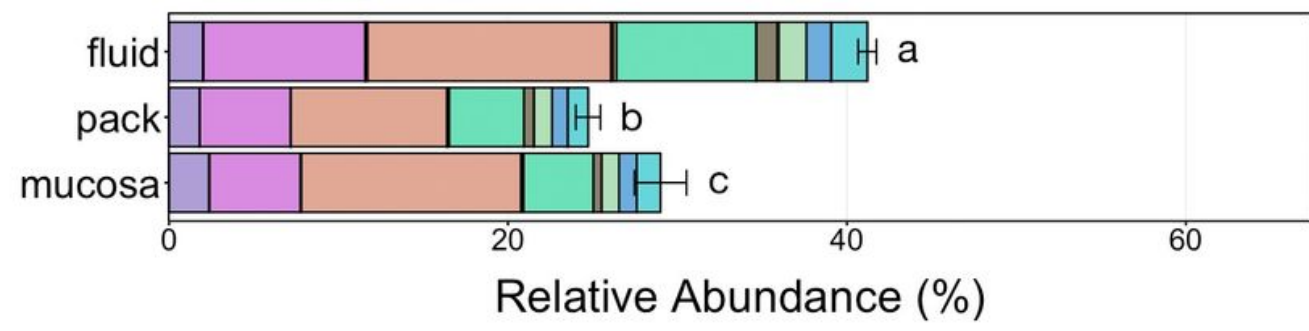

Ruminococcaceae un. Clostridiales Lachnospiraceae Veillonellaceae Erysipelotrichaceae

Figure 6 
Bar plot showing the relative abundances of Bacteroidetes and Firmicutes. Error bars display the standard error of the mean for each phylum and colors represent the relative abundance of families of the two phyla within rumen fluid, pack, and mucosa-associated communities. The top five most abundant families within each phylum are displayed in the legend. Significant differences in the relative abundance of the two phyla are illustrated by different letters (Pairwise Wilcoxon rank-sum with Benjamini-Hochberg correction, $p<0.05, n=12$ ). Abbreviations: un., unclassified

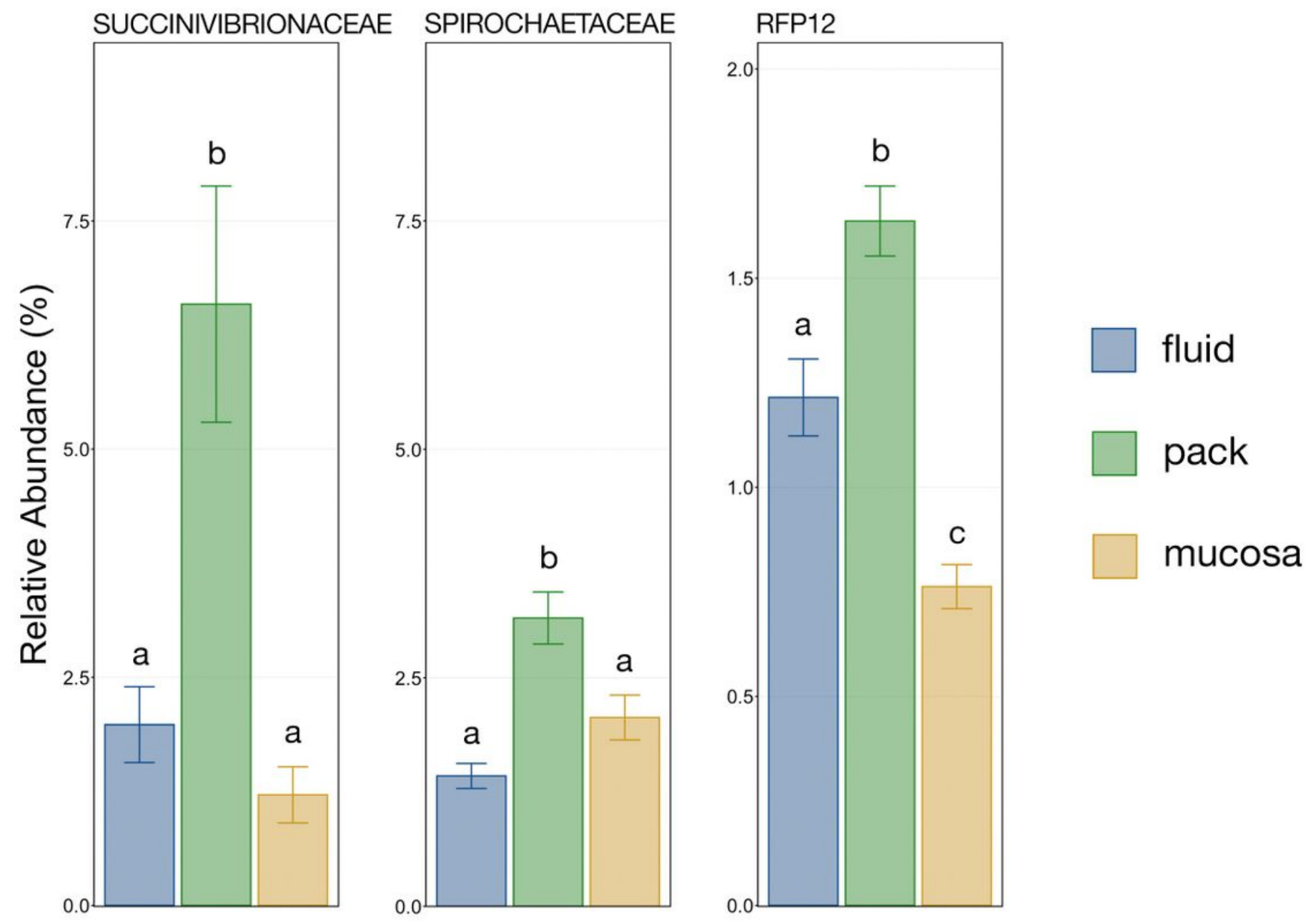

Figure 7

Barplot showing the relative abundances of pack-discriminant taxa. Error bars display the standard error of the mean of the relative abundance of Succinivibrionaceae, Spirochaetaceae, and RFP12 in fluid, pack, and mucosa-associated communities. Significant differences between the relative abundances of each family are illustrated by different letters (Pairwise Wilcoxon rank-sum with Benjamini-Hochberg correction, $p<0.05, n=12$ ).

\section{Supplementary Files}

This is a list of supplementary files associated with this preprint. Click to download. 
- SIPinnellRumenSciRep.docx

Page 20/20 